\title{
LA CLASE SOCIAL COMO CATEGORÍA DIALÉCTICA: HACIA UNA RECONSTRUCCIÓN
}

\author{
Jesús Rodríguez Rojo
}

Universidad Pablo de Olavide, Sevilla

http://dx.doi.org/10.5209/NOMA.56437

\begin{abstract}
Resumen: Ante los cambios que han tenido lugar en las últimas décadas, la revisión y re-lectura de las teorías se convierte en un deber para los investigadores. Este artículo ofrece una interpretación de la categoría de clase social a partir de la lectura en clave lógico-sistemática de El capital de Karl Marx a los 150 años de su primera edición. Para ello, se desarrolla, a partir de la estructura de esta obra, un recorrido por sus principales categorías y su relación con las clases sociales en el modo de producción capitalista. Como final, se apuntan algunos planteamientos esenciales para pensar las clases sociales y la lucha de clases en las formaciones sociales contemporáneas.
\end{abstract}

Palabras clave: teoría marxista, clase social, conciencia de clase, lucha de clases.

\section{The social class as a dialectical category: towards a reconstruction}

Abstract: Due to the changes that have taken place in the last decades, the revision and re-reading of the theories is a must for researchers. This paper tries to offer an interpretation of the category of social class from an analysis in a logical-systematical way of Karl Marx's Capital, 150 years after its first edition. In order to do that, we develop, from this oeuvre's structure, a travel around its most important categories and its relationship with the social classes in the capitalist mode of production. Finally, we point out some essential ideas to think the social classes and the class struggle in the current social formations.

Key words: Marxist theory, social class, class consciousness, class struggle.

\section{Introducción}

En las últimas décadas, sin duda, las ciencias sociales han reconocidos pautas novedosas que tenían lugar en las sociedades contemporáneas. Teorías, de la mano de movimientos, como el feminismo, el colectivo LGTB o el anti-racismo han entrado con fuerza en la sociedad y en los centros de investigación. A su vez, el capitalismo ha experimentado alteraciones que dan lugar a cambios en las estructuras sociales. Con ello, las desigualdades y sus formas "identitarias" han sido reexaminadas de manera casi compulsiva. 
Las teorías clásicas han sido evaluadas una y otra vez y sometidas a severos balances. Seguramente, en el marco de la teoría crítica, el marxismo ha sido una de las teorías más justamente criticada y revisada. De tal praxis surgieron un buen número de propuestas que renegaban del legado inaugurado por Marx para atender a estos cambios sociales. Surgieron planteamientos, en particular sociológicos -Gorz (2005), Bourdieu (2001), Habermas (1987) o Castells (2005) son ejemplos notables-, que partieron de los cambios ocurridos para desacreditar la propuesta clasista y proclamar la necesidad de nuevos paradigmas en los que la centralidad del trabajo da paso a la "semiótica" o a la "construcción cultural". Aunque algunos reconocidos marxistas decidieron en esta coyuntura dar carpetazo a su "etapa marxista" - es el caso de E. Laclau (Laclau y Mouffe, 2006) - y otros, sin abandonar el "marxismo", dejaron de lado la centralidad de las clases y su lucha caso de M. Postone (2007) - la mayoría de la tradición de pensamiento marxista, lejos de titubear, reaccionó con contundencia e incluso beligerancia contra esta tendencia venga por parte de las nuevas tendencias sociológicas (Cf. Valente, 2010, cap. I), de ex-marxistas (Cf. Woods, 1986) o de los propios marxistas (Cf. Carcanholo, 2016).

Nuestro objetivo aquí dista del mecánico restablecimiento de un corpus teórico marxista presuntamente unificado y sólido, propio de los panegíricos del marxismo vulgar; más bien se trata de releer desde la base, sin olvidar las interpretaciones contemporáneas, la teoría marxista en lo que respecta a las clases sociales. Para esta relectura, a la que dedicaremos el apartado más extenso, centraremos nuestra atención en la última obra marxiana, El capital —a los 150 años de la publicación de su primer libro-, y sus borradores, desde una perspectiva lógicosistemática. A partir de los planteamientos de Marx, esclareciendo su naturaleza y alcance, se plantea brevemente el rol de su teoría en el análisis social concreto. No obstante, antes de dicho desarrollo consideramos necesario aclarar algunas ideas básicas en torno a este método.

\section{Cuestiones de método: de lo lógico y lo histórico}

Antes de comenzar la presentación de la propuesta que ocupará estas páginas, es importante situar cuál será el método empleado; pues será precisamente esa cuestión la que hace diferir éste de otros muchos análisis destinados a este mismo propósito.

En este sentido, el primer elemento a destacar es que, dado el carácter preeminentemente ontológico de la obra de Marx —de manera que, como intentamos mostrar en Rodríguez Rojo (2016), las categorías y construcciones teóricas no son esquemas estructurados ad hoc sobre lo real, sino formas ideales de reproducción de la realidad misma-, es en este mismo plano donde debemos situar el debate en relación a las clases sociales. Reducir la "clase social" a un instrumento de análisis de 
la realidad disponible para los científicos significaría renunciar a este nivel de análisis y romper con la naturaleza misma del pensamiento marxiano.

Ahora bien, ¿̇cuál es el método empleado para la determinación ontológica de las categorías empleado por Marx?

Partimos de la idea de que el método empleado en El capital se fundamenta, al menos, en dos tipos de procesos complementarios y relacionados: uno de ellos histórico, que enmarcaría el objeto a partir de su origen, y otro lógico, en el que se desplegarían las determinaciones sincrónicamente. De tal manera que, siguiendo la literatura existente, podemos decir que se trata de un método "históricosistemático" (Lukács, 1974) o "genético-estructural" (Zeleny, 1978). Tales desarrollos, combinados, reflejan la realidad social de la sociedad capitalista y, en particular, de las clases sociales; y a partir de esa aprehensión, puede determinarse su potencialidad histórica: "Se trata de saber lo que el proletariado es y lo que debe históricamente hacer de acuerdo a su ser" (Marx y Engels, 2013: 55). No obstante, la relación concreta entre el desarrollo histórico y lógico ha sido blanco de numerosos problemas, sobre cómo se articulan y relacionan, y de varias propuestas de solución.

La primera, y tal vez más difundida, solución fue la formulada por Engels al identificar el método lógico con "el método histórico despojado únicamente de su forma histórica y de las contingencias perturbadoras" (en Marx, 1980: 340). En la práctica, el "método lógico" quedaba subsumido en el histórico, reduciéndose éste al desarrollo - generalde aquel. Los desarrollos del materialismo histórico quedaron en una posición dominante frente a los del materialismo dialéctico.

En lo que respecta a las clases sociales, la relación entre obrero y burgués es una particularización —una particularización sui generis, sin duda- de un fenómeno casi trans-histórico: la lucha de clases. Así autores como Mandel (1978: 212-216) optan por introducir la lucha de clases como un deus ex machina al despliegue lógico haciendo caso omiso a la diferencia "radical" que, en palabras del propio Marx (1971: 7) existe entre las luchas de clases entre las sociedades "antiguas y modernas". Unas someras e imprecisas consideraciones rotundas respecto a la historia de la humanidad son destacadas en detrimento del desarrollo lógico que tiene lugar en la obra marxiana.

Esta presunta identidad entre la lógica y la historia ha sido explícitamente descartada por Marx (1972: 28-29): "sería impracticable y erróneo alinear las categorías económicas en el orden en que fueron históricamente determinantes". Tal afirmación — junto a la lectura de la obra- nos emplaza en la disyuntiva entre estudiar la clase social como un producto preeminentemente lógico o esencialmente histórico; aquí optamos por el primero. 
Entendemos, pues, que las clases sociales están presentes (siquiera sea de manera latente) desde el primero de los capítulos de El capital y que, a lo largo de la obra, van adquiriendo nuevas determinaciones en relación al despliegue lógico que en esta tiene lugar. Cada "momento" lógico supera al anterior manteniéndolo conteniéndolo en sí en forma de negación. En este sentido, el archiconocido capítulo XXIV ("La llamada acumulación originaria") establecería las bases para un desarrollo sistemático que Marx comienza, de facto, con anterioridad, en el primer capítulo.

En definitiva, partimos de la clase social como una realidad en sí, más allá de la percepción subjetiva que pueda existir por parte de sus miembros o de otros agentes. Pero esta realidad no se inscribe en una totalidad social abstracta sino que se sitúa dentro del modo de producción capitalista, como particularidad histórica y ontológica, y que se aprehende en él a partir de la reproducción lógica (ideal) de su propia estructura.

\section{Desarrollo dialéctico-sistemático de la clase social en El capital}

Para plantear la investigación, partamos de las últimas preguntas que quedan recogidas en los manuscritos que serían editados como el tercer libro de El capital: "La primera cuestión que hay que responder es ésta: ¿qué es lo que constituye una clase? La respuesta resultará automáticamente de la que demos a otra pregunta: ¿qué es lo que convierte a los obreros asalariados, a los capitalistas y a los terratenientes en componentes de las tres grandes clases sociales?" (Marx, 2014, L. III, S. VII: 358).

En las próximas páginas tomaremos muy en serio estos interrogantes en la forma en la que los plantea el autor y a partir del desarrollo que él mismo realiza. Atenderemos, pues, a la recomendación que hace Guerrero (2008: 224): buscar la respuesta, o al menos un camino a ella, volviéndonos hacia el desarrollo anterior del propio Marx.

Para esto, es importante comenzar señalando que, durante los desarrollos que realizaremos, trataremos de reproducir la lógica marxiana y, para ello, es esencial tomar al propio capital no solo como "objeto de investigación", sino también como sujeto del desarrollo (Zeleny, 1974: 297). En el desarrollo ontológico-categorial las diferentes clases sociales se determinan no solo como objetos, como atributos, del capital sino como parte constitutiva de él.

Esto implica que durante el desarrollo que realizaremos estrictamente de la mano de Marx no saldremos del análisis del capital. Profundizaremos en lo que algunos teóricos marxistas clásicos (dos Santos, 1967: 91-94; Carchedi, 1977: 17-21; Wright, 1992: 51-52), en relación a las clases sociales, llamaban el "primer nivel" del concepto de clases sociales: el modo de producción capitalista. No obstante, a diferencia de ellos, 
creemos necesario el empleo de más de un nivel de abstracción, siguiendo la estructura lógica de El capital, para aprehender el conjunto de determinaciones propias de este objeto. Tal es la importancia de la separación —planteada por Rosdolsky (2004) a partir de los Grundrisse y desarrollada, entre otros, por Robles (2014) - entre los "momentos" capital-en-general y varios-capitales; a partir de la cual, como nos disponemos a ver, la clase se constituye como un punto de llegada, no un elemento establecido a priori.

El recorrido por El capital que nos disponemos a realizar no aspira a ser riguroso, es más, rozaría lo obsceno a ojos de un entendido en la materia, sin embargo, nuestro propósito no es explorar el conjunto de determinaciones reproducidas en la obra sino tan solo aquellas que atañan a las clases. Para dinamizar este recorrido dejaremos de lado las citas literales y elementos que serían de crucial importancia si nuestro fin fuera otro: nada exime del estudio del conjunto de la obra si se aspira a ser exhaustivo.

\subsection{De la apariencia en la circulación a la relación entre capital-en- general y trabajo-en-general}

Marx comienza su exposición por la mercancía, donde observa condensadas las primeras determinaciones específicas del modo de producción capitalista. Los bienes que la sociedad reproduce constantemente para satisfacer sus necesidades (son valor de uso) adquieren la capacidad de intercambiarse entre sí (son valor). En tal intercambio, el trabajo (concreto, diverso) invertido en los bienes, mercancías, se vacía de determinaciones (siendo abstracto, homogéneo) posibilitando la igualación de cada mercancía con sus semejantes. La producción, que mantiene su carácter privado y su descoordinación, adquiere una forma directamente social al concurrir ante el conjunto de la sociedad. Dada la asiduidad del intercambio y la descoordinación en la producción (hecho que posibilita la crisis capitalista), se hace imprescindible desdoblar el intercambio - separar "compra" de "venta"-, tal es la función del equivalente general, el dinero. (Marx, 2014, L. I, S. I).

Con el dinero se perfila ya la primera expresión del capital, que solo se introduce, bajo su forma fenoménica, como dinero acrecentado en su propio movimiento, en su mutación (D-M-D'). En este punto, y asumiendo que ni siquiera el dinero como capital es capaz de acudir al mercado sin su particular vehículo, el ser humano, aquel que se encuentra en posesión del dinero-capital se perfila como personificación del capital, como capitalista. Frente a estos poseedores se presentan aquellos que requieren del dinero para subsistir y que pueden trabajar. Entre ambos hacen valer su "complementariedad". La consecuencia directa del intercambio es la inmersión en el proceso de producción. (Marx, 2014, L. I, S. II). 
La igualdad (de valores) expresada en el intercambio queda por completo negada cuando se contempla la capacidad productiva del obrero. La fuerza de trabajo se presenta ahora como valor de uso efectivo, como trabajo creador de valor. Mientras que tan solo los trabajadores aportan valor nuevo al producto, acrecentando su capacidad de intercambio y justificando tanto su salario como la plusvalía; los capitalistas adquieren como determinación esencial el control concreto de las unidades productivas a través de la posesión de los medios de producción empleados para ello. Si los primeros encarnan el trabajo vivo, los segundos hacen lo propio con el objetivado. Sea prolongando o intensificando la jornada de trabajo (plusvalía absoluta), sea potenciando la capacidad productiva -en busca de mayor un rendimiento en cuanto al capital invertido- en sectores de consumo obrero (plusvalía relativa), el capital consigue reproducirse aumentando la razón entre la plusvalía generada y el capital destinado a salarios; aumentando la tasa de explotación. Estos procesos, que no son sino el despliegue de la contradicción entre el carácter simultáneamente privado y social de la producción, son la base del enfrentamiento capital/trabajo. (Marx, 2014, L. I, S. III, IV, V y VI).

La concurrencia de los capitalistas les fuerza constantemente a innovar para aumentar la productividad (las fuerzas productivas, podríamos decir) de sus obreros. El medio fundamental para ello es la maquinización del proceso a través del aumento de la proporción de capital invertido en medios de producción frente a la fuerza de trabajo. El resultado directo de este proceso es, por un lado, la centralización del capital debido a la mayor capacidad de inversión de las empresas más rentables $y$, por otro, la expulsión de una gran parte de la población obrera del mercado laboral - dado que los obreros expulsados no pierden su calidad de oferentes de fuerza de trabajo, esta dinámica conduce al endurecimiento de la competencia inter-trabajo-. Tales son las consecuencias de la ley "general" de la acumulación de capital. (Marx, 2014, L. I, S. VII).

Llegados a este punto, debemos acotar el alcance de las afirmaciones precedentes antes de proseguir. Hasta ahora Marx ha expuesto, ante todo, una realidad, pero no toda la realidad. El capital ha aparecido tan solo como capital-en-general y, frente a él, se ha situado el trabajoen-general. Se ha tratado de exponer el conjunto de determinaciones que afectan a cada capital como capital; podemos decir que se han descrito procesos que tienen lugar por igual en todos los sectores del capital, visto ahora como una totalidad (Marx, 1971: 251, 409). De momento, hemos contemplado la relación de cada capitalista con sus capitalistas semejantes, y lo mismo podríamos decir de los obreros, así como se ha expuesto la relación de los capitalistas con sus obreros. No obstante, nada relaciona aún unos capitales (sectores) con otros. Dicho claro, nada relaciona aún a un obrero metalúrgico con uno cantante 
en tanto que obreros, ni a un capitalista agrícola con uno del sector naval en tanto que capitalistas.

\subsection{Capital como unidad de varios-capitales y clase obrera}

En el libro III de El capital se recogen los últimos pasos dados por Marx para adherir nuevas determinaciones a su estudio de lo concreto. Para ello parte de tomar en consideración la forma inmediata que adquiere la plusvalía para el capital, la ganancia. El capital pierde en ella de vista la fuente de la plusvalía, el trabajo asalariado, y tan solo encuentra una referencia en sí mismo, en el volumen dinerario adelantado. La tasa de explotación se difumina cuando se amalgama, como precio de costo, la inversión en fuerza de trabajo y en medios de producción; la tasa de ganancia, razón entre lo obtenido y lo adelantado, entra en escena. (Marx, 2014, L. III, S. I).

La ganancia, sin embargo, no solo sirve al capital para percibirse a sí mismo, sino también para relacionarse, como capital, con sus semejantes: ahora el capital total de la sociedad aparece como varioscapitales. Esta relación se establece mediante la competencia entre capitales, que ahora se despliega hasta permear el conjunto del capital total. Cuando esto ocurre, cuando los capitales se desplazan para competir entre los diferentes sectores económicos, la tendencia natural es a la igualación de la cuota de ganancia. Los capitales, al desplazarse imperativamente a aquellas ramas más rentables, aumentan la producción y, con ello, terminan rebajando los beneficios hasta llevarlos a la media. Esta tendencia convierte a los capitalistas en una suerte de accionistas del capital total: allá donde inviertan, conseguirán una parte alícuota del conjunto de la ganancia. Los capitalistas encuentran en esta determinación la condición de posibilidad para su constitución como clase; la única base para su solidaridad. (Marx, 2014, L. III, S. II; Marx, 1972: 167-168).

No obstante, en la clase capitalista aparece un elemento de discordia: la renta. Tomaremos, siguiendo a Marx, la renta de la tierra como base para la explicación aunque no se reduce a ella este fenómeno. No nos referimos en este caso a la renta obtenida por la mejor capacidad productiva de unas tierras frente al resto (la renta diferencial), sino a la que emana directamente del control de recursos escasos no reproducibles -o difícilmente - por el trabajo humano (la renta absoluta). Este carácter, si se quiere "monopolístico", existente en algunos sectores se interpone como un límite a la competencia que restringe la capacidad de inversión del capital. Aparece con ello un cisma en la clase capitalista: la capacidad del terrateniente de apropiarse de una parte de la ganancia por el hecho de poseer tales recursos hace que estos posean determinaciones específicas - sin perder su condición de capitalistas - frente a la burguesía, que carece de tal prebenda. (Marx, 2014, L. III, S. VI). 
Recordemos un momento la tendencia de los capitales - en su carrera por alcanzar ganancias extraordinarias - aumento proporcional del capital invertido en medios de producción (capital constante) respecto al invertido en fuerza de trabajo (capital variable) como proceso inherente a la propia dinámica de acumulación. Al articular esto con la recién mencionada tasa de ganancia vemos que el capital como un todo, a partir de la acción de sus partes, pierde cada vez mayor rentabilidad por el hecho de que tan solo la fuerza de trabajo produce el valor. Esta es la conocida, y polémica (Cf. Katz, 2014, cap. IV), ley de la tendencia decreciente de la tasa de ganancia. La consecuencia directa es que, si se aspira a mantener la cuota de ganancia, el capital se ve forzado a aumentar constantemente la tasa de explotación para tratar de compensar una tendencia irrefrenable. Es importante aclarar que dada la constante reorganización del capital que iguala la tasa de ganancia, este aumento no se da en tal o cual rama sino en el capital como un todo. Esta dinámica configura las bases materiales del sujeto colectivo explotado, la clase obrera; un sujeto que surge, sin embargo, como predicado del capital. Tal es concreción de la archiconocida contradicción entre el desarrollo de las fuerzas productivas y las relaciones sociales de producción. (Marx, 2014, L. III, S. III).

\section{Lucha de clases: de la fuerza de trabajo a la clase-para-sí}

Las clases sociales, como es bien sabido, se relacionan entre sí a partir del mutuo enfrentamiento. Tan solo a partir de esta relación específica las clases se reconocen y se constituyen, en este proceso se alcanzan determinaciones no esbozadas hasta ahora: el correlato en la conciencia del desarrollo del capital.

Para realizar este desarrollo procuramos un esquema -Figura 1- a partir del cual podamos resumir el desarrollo que hasta ahora se ha realizado señalando además algunos de los conceptos que desarrollaremos en este apartado: la lucha de clases y su reflejo en la conciencia: 


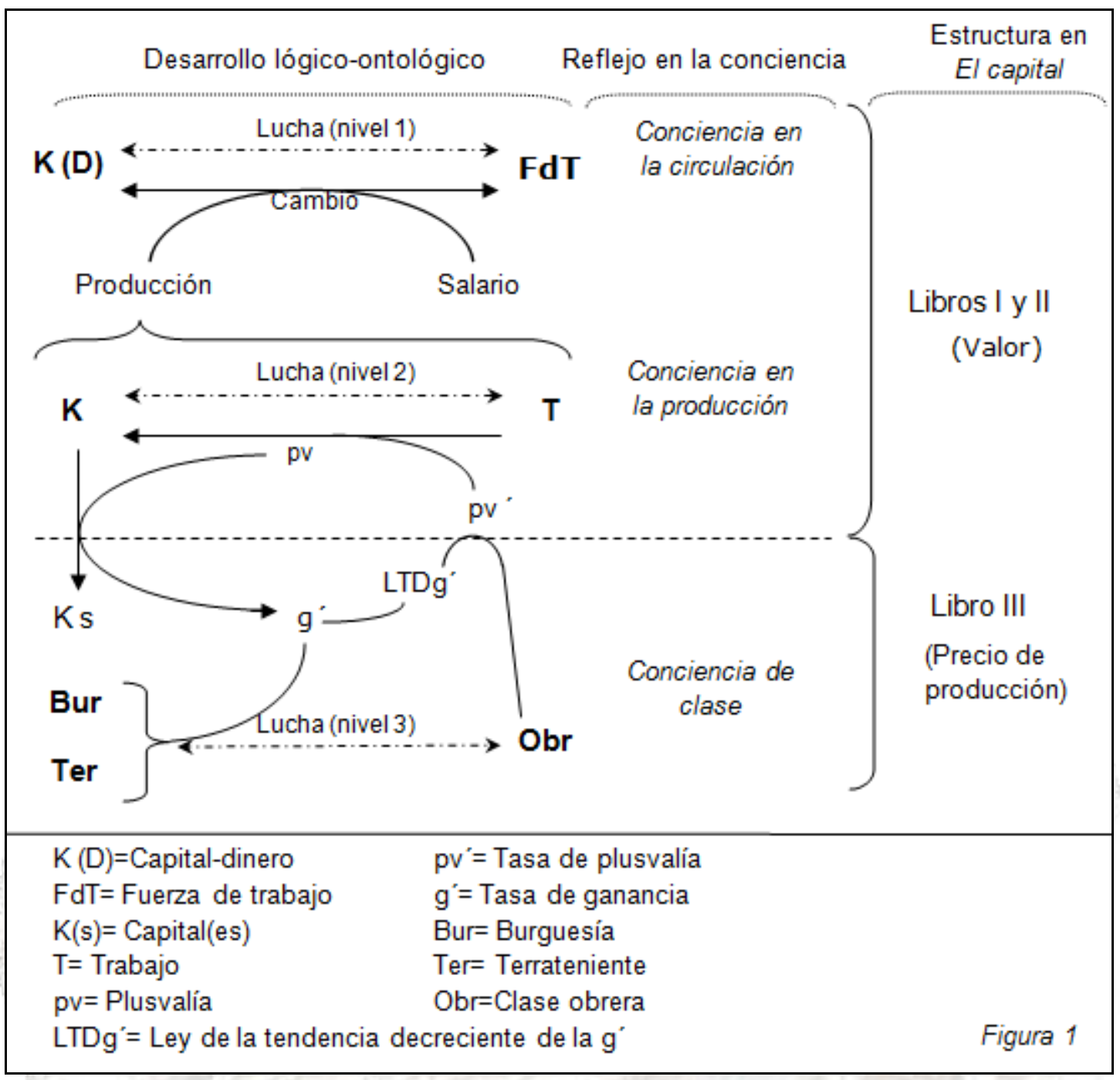

En el esquema se aprecia el desarrollo realizado hasta ahora sin pretender ser especialmente rigurosos en relación a los pormenores de dicho proceso y menos aún en relación a la estructura de El capital. No obstante, en él sí se observan los elementos fundamentales que llevan a constituir las tres clases principales del modo de producción capítalista y su relación conflictiva. Para comprender esta relación se apunta ya en el esquema la existencia de tres "niveles" de lucha de clases y su reflejo en la conciencia que procedemos a explicar.

La primera relación - nivel 1 - que se establece es una relación de compra-venta donde el capital en su forma dinero se enfrenta con la fuerza de trabajo. Aquí, comprador y vendedor se enfrentan, mutatis mutandis, como en cualquier otra relación de compraventa (ni que decir tiene que esta relación se constituye de manera asimétrica debido a la desposesión del potencial trabajador y la capacidad de compra del capital). Cada uno de los contrarios aspira a optimizar el intercambio, a inclinarlo a su favor todo lo posible: el salario, la intensidad o el tiempo de trabajo son factores que se determinan en tal relación. Esta lucha se manifiesta de manera colectiva, no sin las trabas 
impuestas por la competición intra-clase; en lo que respecta a la fuerza de trabajo la forma que asume es el sindicato, mientras que para el capital se concreta en la patronal. El resultado "normal" de este proceso es la venta de la fuerza de trabajo por su valor, permitiendo así la reproducibilidad de la misma; y también del capital.

En este momento la conciencia del trabajador se enfrenta a la conciencia del capitalista como personificaciones de mercancías, como apéndices de las mismas, una de ellas elevada a "capital", el dinero, la otra simple mercancía, la fuerza de trabajo. Las conciencias se enfrentan como enajenaciones. El trabajador y el capitalista carecen en ese momento del control (hacia fuera) de sus propias relaciones, que se muestran de manera fetichizada en el precio (en este caso de la fuerza de trabajo). En términos clásicos, se trata de una conciencia "tradeunionista" (por ambas partes) que trata de mejorar sus condiciones para fijar un valor de cambio favorable.

El resultado del primer nivel de lucha es la incorporación del trabajo a la producción, en el capital-en-general. Como señalamos anteriormente, tan solo desde esa premisa puede comprenderse el desarrollo del capital con el trabajo, que es una forma de relación del capital consigo mismo, en la producción. Es en ella donde el trabajo como potencia produce el valor no solo para cubrir su propio salario y los medios de producción, sino también para la acumulación del capital, la plusvalía. Las personificaciones del capital aparecen aquí como detentores del control de la producción - que asume la forma de propiedad privada de los medios de producción - en cada una de las unidades productivas. En esta relación se constituye la posibilidad revolucionaria del trabajo-en-general: la aspiración a romper la frontera entre producción y control. Antes hemos visto como su condición de "valor" otorgaba a la mercancía fuerza de trabajo determinaciones que la hacían entrar en la lucha de clases; ahora, el trabajo-en-general, vivo, "valor de uso" para el capital, se constituye como sujeto, y de ahí la potencialidad negativa del mismo (Marx, 1971: 215; Marx, 1980: 27.4-276) - lo que no implica inmediatamente una capacidad afirmativa respecto a un "post-capitalismo"- - Si la clase obrera defenta una posición ontológicamente esencial en un sentido revolucionario es, por tanto, debido a su condición de capital (variable), gozando este de una posición realmente privilegiada como "potencia económica, que lo domina todo, de la sociedad burguesa" (Marx, 1971: 28). Solo asumiendo esa capacidad negativa inmanente al capital se puede emplear la expresión hegeliana de "negación de la negación" (Marx, 2014, L. I, S. VII: 258).

La conciencia del obrero, reducido a trabajo-en-general, se enfrenta ahora a su propia potencia de manera enajenada, personificada en el capitalista para el que trabajo. Esta situación no hace sino acentuarse con la formación del obrero colectivo y el sistema de máquinas; en él, descubre, por un lado, en los medios de trabajo (la maquinaria es el 
caso paradigmático) su condición de apéndice del proceso que protagoniza y, por otro, en la rotación de los objetos de trabajo, su conciencia de espectador. Ni siquiera los obreros con mayor conciencia del proceso productivo concreto escapan de su enajenación debido a su crónica incapacidad para apropiarse de tal proceso. Su práctica productiva queda por completo fuera de su control (ahora también hacia dentro de la producción social). En este marco puede entenderse la voluntad de la conciencia por apropiarse de su capacidad productiva. Como apunta D. Foley (1986: 91), el trabajo-en-general no encuentra reflejo alguno en su conciencia de la extracción de la plusvalía; solo encuentra, mediada por las dinámicas de acumulación del capital y su conciencia en la circulación, la conciencia de su impotencia frente a las fuerzas del capital. Se percata de la imposibilidad de conciliar la apropiación de su potencia productiva con la acumulación del capital, es una conciencia de la propia alienación (hacia dentro y hacia fuera del proceso de producción). La conciencia revolucionaria no emana de la ausencia de enajenación, sino de la consciencia de la amplitud de esta enajenación; para Marx y Engels (2013: 54) la cuestión era la producción de "la miseria consciente de su miseria moral y física, el embrutecimiento consciente de su embrutecimiento y que, por esta razón, tratan de suprimirse a sí mismos". Por otro lado, los capitalistas no experimentan en este punto ningún salto cualitativo en su conciencia. Ellos sí controlan directamente sus unidades productivas aun manteniendo el descontrol previo hacia el exterior, en relación al mercado, respecto al cual actúan de manera más o menos reactiva. Ya desde las primeras determinaciones se agota la capacidad superadora de los capitalistas en relación al modo de producción: son, a todos los efectos, una clase-para-el-capital. En lo inmediato, cada componente de la clase presenta una conciencia de "su" capital, que se expresa como "éxito" o "fracaso" de la estrategia particular en relación a la de los competidores; tal es la condición de ser depositaria de la restringida subjetividad de un fragmento de capital.

Como se podría imaginar, el tercer, y último, nivel de lucha deviene de la situación creada por la transformación de la plusvalía en ganancia. El establecimiento de la tasa general de ganancia divide sin duda a la clase capitalista, genera tensiones entre los concurrentes en marcos libres de "barreras" y los disponen de dichas restricciones; y, sin embargo, no hay una relación contradictoria entre ellos. No debemos olvidar la naturaleza netamente capitalista del terrateniente en el agro moderno. El terrateniente $-U$ otro tipo de rentista- no es, entonces, ninguna suerte de resquicio del feudalismo; es un capitalista más. Las diferentes personificaciones del capital se encuentran unidas por un elemento común, el control de la producción que se refleja en una ganancia determinada, y, a la vez, encuentran elementos de conflicto debido a la capacidad de algunos de apropiarse de rentas inaccesibles al resto. 
La lucha adquiere determinaciones de nuevo como reacción y parte del capital. La caída de la tasa de ganancia obliga a la multiplicidad de capitales a aumentar la explotación de la clase obrera. Ahora bien, esta caída de la tasa de ganancia no se manifiesta de manera lineal, adquiere la forma de crisis generales y recurrentes del capital, en las cuales el capital se ve forzado a aumentar de manera contundente la explotación, para lo que no puede recurrir al incremento del rendimiento productivo que devalúe los medios de consumo de los obreros (plusvalía relativa) y se ve forzado a la dilatación de la jornada laboral o la intensidad del trabajo (plusvalía absoluta), así como al pago de los salarios bajo su valor. Por tanto, la constitución de la clase obrera como tal se forma también de manera progresiva, desigual e interrumpidamente a través del enfrentamiento con el capital. En este sentido, para nosotros, el debate en relación al derrumbe inevitable del capital o la revolución (Cf. Mattick, 2014, cap. III) encuentra el principal problema en su propio planteamiento: la revolución es inmanente al capital, no una exterioridad, y se produce sobre la base de las crisis propias del modo de producción capitalista.

Esta secuencia interrumpida se manifiesta también en la formación de la conciencia de clase, a través de una acumulación de experiencia. Es en este momento cuando los trabajadores, en la lucha, pueden reproducir en su conciencia el proceso de constitución de sí mismo como una clase, y, con ello alcanzar el estatus de clase-para-sí (Marx, 1973: 243), de clase efectiva. La situación común, conciencia del doble descontrol del proceso productivo (hacia fuera y hacia dentro), expresada en la experiencia compartida, se establece como base sobre la que se erigen los intereses comunes de la clase obrera y su capacidad de actuar conjuntamente. No huelga apuntar que, en relación a la famosa polémica Thompson-Anderson, si bien la clase, para constituirse como clase, necesita - como plantea Thompson (1984: 38)- de una experiencia colectiva, esas experiencias devienen de una condición primaria, a saber, personificar una mercancía determinada en el intercambio. Tal elemento es lo que da sustento a la propia clase social en Marx. Por tanto, la clase es objetiva - como apunta Anderson (2012: 47) - en tanto la condición de mercancía lo es.

\section{Mediaciones: de la totalidad del capital a la totalidad concreta}

La exposición que hasta ahora ha tenido lugar se ha acotado a diferentes niveles de abstracción relativamente elevados. En la realidad, claro, estas categorías, las clases, no se presentan siempre directa 0 inmediatamente. Para acercarnos a ella en lo concreto, requerimos de un desarrollo posterior, labor a la cual dedicaremos este apartado. Se trata de esbozar la relación entre el modo de producción capitalista (totalidad del capital) y el resto de la totalidad social concreta. Esta es la relación que, en la obra de Marx, se establece entre los escritos 
"económicos", El capital, y los "periodísticos", El 18 brumario de Luis Bonaparte, por ejemplo.

Como ya apuntamos, no todas las ocasiones en las que Marx emplea la palabra "clase" se refiere a lo mismo. La noción restrictiva que hemos planteado hasta ahora contrasta con visiones más amplias que también existen en su obra. Por ejemplo, propiamente en El capital, Marx dedica algunas palabras a describir el "lumpen-proletariado", los estratos más degradados de la población obrera, ¿constituye una nueva clase? Tal vez. Sin embargo no se trata de una clase del modo de producción capitalista (aun siendo producida por este) en el sentido recién expuesto; pues no se relaciona de manera constitutiva con el capital, al menos aquellos que son, para Marx (2014, L. I, S. VIl: 111), el "peso muerto del ejército industrial de reserva". Y es que, aunque ésta clase sin duda se encuentra en la totalidad social, no forma parte de la totalidad que compone el modo de producción capitalista. En realidad, entre la clase obrera tal como se ha expuesto y una más adecuada al análisis de coyunturas en espacios concretos (caso de El 18 brumario...) existe una diferencia en relación al nivel de abstracción empleado. Este salto requiere la consideración de una estructura de mediaciones - véase Netto (2011) para una introducción a conceptos como "mediación" o "totalidad"- que trataremos de esbozar en estas líneas.

El modo de producción no se presenta inmediatamente. Existen elementos que intervienen en la manera en que este se manifiesta y no solo de manera esporádica, sino sistemática y estructuralmente. El sexo, la edad o la raza, por ejemplo, generan notables diferencias dentro de las clases pero, para explicar estas diferencias no podemos sino ver las relaciones del modo de producción capitalista con el sexismo, racismo o sistema etario en su particularidad. Podríamos explicar esta necesidad de estudiar estos sistemas, por ejemplo, diciendo que si bien la lógica de la acumulación explica la necesidad de pagar menores salarios a los colectivos en peores circunstancias sociales, no explica por qué son, por ejemplo, las mujeres, los negros o los jóvenes las que se sitúan en dicha posición. Al igual que del sexismo o del racismo, la aprehensión de la estructura de mediaciones debe dar cuenta de los trabajadores no subsumidos en los capitales individuales sino empleados por cuenta de la administración de los intereses del capital social, el Estado capitalista. Aunque no es nuestro propósito aquí centrarnos en este tema, creemos fundamental tener en cuenta este factor a la hora de elaborar la mencionada estructura de mediaciones.

¿Cómo debe plantearse el sistema de mediaciones en relación a la expresión de las clases sociales? Responder a esta pregunta ha sido y continúa siendo uno de los retos de la tradición marxista hoy. No se trata de un terreno baldío: existen estrategias dignas de consideración.

La primera, hoy muy en boga, es la del pensador italiano A. Gramsci. El concepto de "bloque histórico" ha sido especialmente recurrente a la 
hora de denotar aglutinamientos de grupos en torno a una clase, asumiendo este un papel dominante o hegemónico. La autonomía de la esfera política respecto de la economía —reduciendo su vinculación a un fenómeno "tendencial"- - y la relevancia que recibe dicha esfera - a la hora de, por ejemplo, constituir el bloque histórico- han hecho de su planteamiento una alternativa para el "mecanicismo" presente en el marxismo (Gramsci, 1974: 277, 408-409; Portelli, 1977). Sin duda, la aspiración de Gramsci no era otra que dar con un sujeto histórico, que pudiera superar el capitalismo y, para ello, recurre a la política, dotando a las "clases subalternas" de un instrumento de articulación, el bloque histórico, donde se amalgaman los sectores. Esta concesión proporciona la posibilidad de comprender acontecimientos concretos de la lucha política, pero acaba por perder la vinculación con las determinaciones económicas; tales determinaciones entran en su planteamiento tan solo de manera "histórica". La economía queda, en él, indisolublemente ligada al "filosofismo historizante" (Lefebvre, 1971: 169). El capital pierde por completo su condición de sujeto en su mirada histórica, recayendo esta condición en los conglomerados ideológicamente formados de las "clases subalternas".

La propuesta gramsciana en realidad obedece a una dinámica ajena al pensamiento de Marx, a saber, establecer una suerte de continuum en cuyos extremos se encontrarían los grupos sociales peor y mejor situados "económicamente". Así el "precariado" -para una definición del concepto, véase Braga (2014: 30) - quedaría a la izquierda de la mayoría de la clase obrera que a su vez estaría a la izquierda de la "aristocracia obrera"; esto daría paso a los estratos inferiores de la administración pública, que a su vez serían seguidos por la pequeña burguesía y terminaría, tal vez, con la gran burguesía monopolista. Sería a partir de una escala de este tipo que se formarían las alianzas de clases (Poulantzas, 1973: 104). En esta lectura los trabajadores del Estado caerían de un lado u otro según en función de su "modo de vida" o su "papel político" (Poulantzas, 1973: 113). A una posición similar llegan los neomarxistas partiendo de una matriz de diferentes explotaciones (Wright, 1992: 65-75), u otras aportaciones recientes aún más elaboradas (Piqueras, 2007). No podemos rechazar de plano conclusiones de estos trabajos, pero sí pensamos que devienen de una praxis extraña a la que hemos observado en El capital. La articulación estructurada en base al despliegue categorial se reemplaza por una definición -más o menos explícita; más o menos desarrollada- de "clase" y, acto seguido, se procede a "complejizarla" mediante nuevas definiciones que entran a ofrecer dimensiones del análisis; tal es el modus operandi de lo que en otra ocasión llamamos "sociología representativa" (Cf. Rodríguez Rojo, 2016).

Una manera alternativa de proceder es de la que surge el concepto de "clase-que-vive-de-su-trabajo" que formula R. Antunes (2000, cap. VI). En él, a partir de la influencia de Lukács y su escuela-Mészáros en 
concreto- el trabajo recibe la capacidad de articulación de la heterogeneidad de la formación social capitalista. El trabajo en el aparato estatal, el trabajo doméstico o el "sub-proletariado" quedarían incluidos, subsumidos, en esta categoría; mientras que se excluyen las actividades de aquellos que detentan el control de los procesos de trabajo como lo ejecutivos o altos funcionarios (Antunes, 2000: 103-104). La propuesta de Antunes no solo contribuye, sin duda, a reavivar el debate a partir de una base sólida, sino que lo hace asumiendo el desarrollo de Marx como punto inequívoco de partida. Además, a diferencia de Gramsci, realiza una definición mucho más "objetiva", sin recurrir al discurso político como aglutinador. No obstante, para nosotros, esta categoría concreta tan solo es compatible con el corpus marxiano si es planteada como fruto de las mediaciones inherentes a la totalidad social y no (como en ocasiones el texto da a entender) como una actualización del concepto de clase. No creemos que la categoría clase obrera tenga hoy diferente valía que en tiempos de Marx aunque, sin duda, ésta se expresa de manera diferente, y es de esa diferencia de expresión de la que hay que dar cuenta.

Las propuestas esbozadas son sanos intentos por actualizar y desplegar la teoría marxista de las clases sociales. Tanto el "bloque histórico" como la "clase-que-vive-de-su-trabajo" parecen intentos por ampliar el sujeto revolucionario para aprehender una realidad que, como apuntábamos al comienzo, se percibe como diversa. Aunque los esfuerzos son notables y tienen la virtud de generar conceptos aplicables empírica o normativamente a la realidad, en su proceder se percibe una ruptura con la estructura de Marx: se parte de una definición simple -que no da cuenta de las determinaciones apuntadas en este documento-de clase obrera como sujeto revolucionario para considerarla como restringida y aportar un concepto más o menos novedoso que, con sú enunciación, superaría este déficit.

Consideramos que si, por el contrario, se pretendiera continuar la vía de Marx debería evitarse este salto del modo de producción a la totalidad y escalonarlo en diferentes niveles que aporten determinaciones a lo concreto. Por ejemplo, una vez comprendemos la tasa general de ganancia y la lucha de clases necesitamos enfrentarnos al Estado capitalista, siendo este para nosotros la forma nacional de metabolización de ambos fenómenos que, en conjunción, representan la unidad del capital social -siendo el Estado "el capitalista colectivo ideal" (Engels, 1975: 339)—; así, los empleados del estado no serían más que los empleados por cuenta de la administración de los intereses del capital en su forma nacional. Igualmente, podríamos abordar el controvertido tema trabajo reproductivo no remunerado; este trabajo conecta a sus realizadores (generalmente realizadoras) con la cualidad de mercancía del cónyuge (o familiar) al ser este su mediación con la "relación social general", la mercantil-capitalista (Caligaris, 2013: 83-84); esto ocurriría de tal manera que se expandirían las determinaciones de 
clase determinaciones de clase, al menos una parte de ellas, a su unidad de reproducción, su familia. Valga esta telegráfica exposición de ejemplos - que deben ser discutidos y desarrollados- como indicación de una dirección a seguir, la de desarrollar un proceso de aprehensión cuyo objetivo sea el agotamiento de las determinaciones del modo de producción capitalista. Para ello, es preciso comprender el modo de producción capitalista en relación, como apuntábamos, con otras dinámicas coexistentes y articuladas con él. En relación a ese camino, que bien merece ser recorrido, este documento no pretende ser más que un emplazamiento algo más consistente de los primeros jalones.

\section{Conclusiones}

Creemos que, a lo largo del desarrollo de estas líneas se ha demostrado la verosimilitud de las tesis que se desprenden de lo desarrollado en relación a nuestra premisa, a saber, que en el análisis marxiano de las clases sociales el desarrollo lógico-sistemático de la especificidad histórica adquiere un papel dominante. El estudio marxista de las clases sociales, como clases constitutivas del capital, requiere de un acompañamiento sistemático de los desarrollos de los tres libros de El capital. Los planteamientos que se realizan en tal obra y, sobre todo, la estructura que de ella emana, constituyen un marco rico para desarrollar el análisis de las clases sociales. Los diferentes niveles de abstracción que se plantean, aceptando como premisa el carácter lógico-ontológico de la obra de Marx, no pueden sino representar en realidad niveles de realidad social y, como tales, deben ser estudiados y desarrollados, no por separado sino conjuntamente, de manera dialéctica. De ahí que el propio Marx (1982: 151) considerara su obra como un "todo artístico", integrado.

Todo ello, sin embargo, no es suficiente, ni puede serlo, para enfrentar la realidad; no solo por los cambios que han tenido lugar en las últimas cinco décadas, sino por la complejidad misma de las sociedades. Para ello es imprescindible el estudio riguroso de las estructuras de mediaciones que operan desde el modo de producción capitalista y que modifican su manifestación en las acciones colectivas. Aunque, la parte más importante de la obra de Marx se restrínja al estudio del modo de producción capitalista, se puede conciliar los planteamientos que de él emanan con otros que, en conjunto, sirvan para describir fielmente las sociedades modernas. Esto requiere, al menos, de seguir dos caminos comunicados pero no idénticos: el desarrollo del análisis del modo de producción capitalista como una totalidad articulada a través del la formulación de nuevas determinaciones que aproximen el análisis a lo concreto y el estudio de fenómenos sociales diferentes al propio capital en la medida en que lo sean (entre los que se incluyen otros tipos de dominación). Proseguir por estos caminos críticamente es 
el reto más sustantivo al que los marxistas se han enfrentado desde hace décadas; este trabajo ha pretendió regresar establecer las bases de esos caminos desde una perspectiva dialéctico-sistemática en lo que se refiere a un problema crucial: las clases sociales.

Por último, concluimos resumiendo los principales resultados alcanzados en los siguiente enunciados: (1) las clases sociales se constituyen no como un a apriorismo sino en el desarrollo de un objeto-sujeto históricamente específico: el capital; (2) el análisis de las clases sociales parte de la condición de personificación de mercancía y se desarrolla como contraparte inmanente al propio capital a través de la lucha clasista hasta constituirse como clase "para si"; (3) el desenlace del análisis de Marx se constituye como un punto de partida para desarrollos ulteriores que den cuenta de la totalidad social; (4) tales desarrollos, si aspiran a ser consecuentes con el método empleado, deben evitar enfrentarse directamente a la totalidad social, reproduciendo la estructura de mediaciones a través de niveles de abstracción.

\section{Bibliografía}

Anderson, P. (2012) Teoría, política e historia. Madrid: Siglo XXI.

Antunes, R. (2000) Os sentidos do trabalho. São Paulo: Boitempo.

Bourdieu, P. (2001) Poder, derecho y clases sociales. Bilbao: Desclee de Brouwer

Braga, R. (2014) "Precariado e sindicalismo no Brasil contemporâneo: Um olhar a partir da indústria do call center" Revista Crítica de Ciências Sociais, 113, 25-52.

Caligaris, G. (2013) "Un balance crítico de la teoría marxista y neomarxista de las clases sociales", en E. Chávez (ed.) en Desigualdad y movilidad social en el mundo contemporáneo (pp. 71-87). Buenos Aires: Imago Mundi.

Carcanholo, M. D. (2016) "Algumas implicações da exasperação historicista da teoria do valor de Marx por Moishe Postone" Marx e o marxismo, 7 (4), 303-317.

Carchedi, G. (1977) On the economic identification of social classes. Londres: Routledge Direct.

Castells, M. (2005) La era de la información: la sociedad en red. Madrid: Alianza. 
Dos Santos, T. (1967) "El concepto de clases sociales" Anales de la Universidad de Chile vol. 1 (141-144), 81-116.

Engels, F. (1975) Anti-Düring. La Habana: Pueblo y educación.

Foley, D. (1986) Understanding Capital. Cambridge: Harvard University Press.

Gorz, A. (2005) O imaterial - Conhecimento, valor e capital. São Paulo: Anna Blume.

Gramsci, A. (1974) Antología. Madrid: Siglo XXI.

Guerrero, D. (2008) Resumen completo de El capital de Marx. Madrid: Maia.

Habermas, J. (1987). Teoría de la acción comunicativa (I). Madrid: taurus.

Katz, C. (2014) La economía marxista hoy. Madrid: Maia.

Laclau, E. y Mouffe, C. (2006) Hegemonía y estrategia socialista. Buenos Aires: FCE.

Lefebvre, H. (1971) O fim da historia. Lisboa: Dom Quixote.

Lukács, G. (1965) Estetica. I (1). Barcelona: Grijalbo.

Mandel, E. (1978) Tratado de economia marxista. I. Lisboa: Livraria Bertrand.

Marx, K. (1971) El 18 brumario de Luis Bonaparte. Barcelona: Ariel.

Marx, K. (1971) Elementos fundamentales para la crítica de la economía política. Grundrisse. I. Madrid: Siglo XXI.

Marx, K. (1972) Elementos fundamentales para la crítica de la economía política. Grundrisse. II. Madrid: Siglo XXI.

Marx, K. (1973) Miseria de la filosofía. Madrid: Aguilar.

Marx, K. (1980) Contribución a la crítica de la economía política. México DF: Siglo XXI.

Marx, K. (2014) El capital. Crítica de la economía política. Madrid: Akal. [L.: libro; S.: sección]

Marx, K. y Engels, F. (1983) Cartas sobre "El capital". La Habana: Editorial política.

Marx, K. y Engels, F. (2013) La sagrada familia. Madrid: Akal.

Mattick, P. (2014) Crisis económica y teorías de la crisis. Madrid: Maia.

Netto, J. P. (2011) Introdução ao estudo do metodo de Marx. São Paulo: Expressão popular.

Piqueras, A. (2011) "Desafíos del Trabajo como sujeto histórico en el capitalismo tardío declinante", en A. Piqueras \& W. Dierckxsens (eds.) El colapso de la globalización (pp. 207-278). Madrid: El viejo topo. 
Portelli, H. (1977) Gramsci y el bloque histórico. Madrid: Siglo XXI.

Postone, M. (2007) Marx Reloaded. Madrid: Traficantes de sueños.

Poulantzas, N. (1973) "Las clases sociales", en Las clases sociales en América latina (pp. 96-126). México DF: Siglo XXI.

Poulantzas, N. (1978) Poder político y clases sociales en el estado capitalista. Madrid: Siglo XXI.

Robles, M. (2014) "Sobre algunos momentos del concepto de capital", en M. Robles (ed.) Dialéctica y capital (pp. 383-447). Buenos Aires: RyR.

Rodríguez Rojo, J. (2016) "La sociología representativa contra El capital. Los retos de la dialéctica y la crítica de la epistemología" Encrucijadas, 12 , a 1203.

Rosdolsky, R. (2004) Génesis y estructura de El capital de Marx. Madrid: Siglo XXI.

Thompson, E. P. (1984) Tradición, revuelta y conciencia de clase. Barcelona: Crítica.

Valente, J. (2010) Classes, valor e acção social. Lisboa: Página a pagina. Wood, E. M. (1986) The retreat from class. Londres: Verso.

Wright, E. O. (1992) "Reflexionando, una vez más, sobre el concepto de estructura de clases", en J. Carabaña y A de Francisco (eds.) Teorías contemporáneas de las clases sociales (pp. 17-126). Madrid: Zona abierta.

Zeleny, J. (1978) La estructura lógica de "El capital" de Marx. Barcelona: Grijalbo. 